\title{
Self-derivations on the noncommutative Schwartz space
}

\author{
Krzysztof Piszczek ${ }^{1}$
}

Received: 19 April 2016 / Accepted: 25 October 2016 / Published online: 10 November 2016

(C) The Author(s) 2016. This article is published with open access at Springerlink.com

\begin{abstract}
We characterize derivations acting on the noncommutative Schwartz space.
Keywords (Noncommutative)Schwartz space · M-convex (Fréchet) *-algebra $\cdot$ PLS-space . Bi-module · Derivation · Amenable
\end{abstract}

Mathematics Subject Classification Primary 47B47 · 47L10 · 46A13; Secondary 46K10

\section{Introduction}

The aim of the paper is twofold. First, we give a full proof of the characterization of boundedly approximately contractible Fréchet algebras. This result was announced (without proof) in [24]. Second, we apply this characterization to show that if $\delta: \mathcal{S} \rightarrow \mathcal{S}$ is a derivation acting on the noncommutative Schwartz space $\mathcal{S}$ then there is an element in the multiplier algebra of $\mathcal{S}$, say $x$, such that $\delta(a)=a x-x a$ for all $a \in \mathcal{S}$. Versions of for the so-called smooth *-algebras-see [10, Section 4.8, Proposition]. To prove our derivation result we will need to rely at some point on the nuclearity (in the sense of Grothendieck) of the multiplier algebra of $\mathcal{S}$. Therefore we may say that, in a sense, all self-derivations on the noncommutative Schwartz space are inner. This result may be deduced from [25, Prop. 6.3.2]. The method of proof in [25] uses the theory of the so-called $\mathcal{O}^{*}$-algebras. Our approach will necessarily be different.

The noncommutative Schwartz space (the definition of which is given in the next section) is a specific $m$-convex Fréchet *-algebra. It is isomorphic (as a Fréchet *-algebra) to the algebra $L\left(\mathcal{S}^{\prime}(\mathbb{R}), \mathcal{S}(\mathbb{R})\right.$ ) of linear and continuous operators from the space of tempered distributions into the Schwartz space of smooth rapidly decreasing functions (for more representations see [8, Th. 1.1]). It is also isomorphic (as a Fréchet space) to $\mathcal{S}(\mathbb{R})$.

Krzysztof Piszczek

kpk@amu.edu.pl

1 Faculty of Mathematics and Computer Science, Adam Mickiewicz University in Poznań, ul. Umultowska 87, 61-614 Poznań, Poland 
The noncommutative Schwartz space and the space $\mathcal{S}(\mathbb{R})$ itself play a role in a number of fields, e.g.: structure theory of Fréchet spaces and splitting of short exact sequences-[18, Part IV]; K-theory-[1,6,15,19]; $C^{*}$-dynamical systems-[13]; cyclic cohomology for crossed products-[26]; locally convex analogues of operator spaces-[11,12]; quantum mechanics, where it is called the space of physical states and its dual is the so-called space of observables-[9]. Recently, some progress in the investigation of the noncommutative Schwartz space has been made. This contains: functional calculus-[4]; description of closed, commutative, ${ }^{*}$-subalgebras-[3]; automatic continuity-[22]; amenability properties-[20, 22]; Grothendieck inequality-[17]. Specifically, the multiplier algebra of the noncommutative Schwartz space has been identified and investigated in [5].

The paper is divided into four parts. In the next section we collect all the necessary definitions and basic properties of the objects involved. Section 3 contains a characterization of boundedly approximately contractible Fréchet algebras. In the last section we use this characterization (more precisely, the proof of it) to show the main result of the paper, i.e. we prove that if $\delta: \mathcal{S} \rightarrow \mathcal{S}$ is a derivation on the noncommutative Schwartz space then there exists an element $x$ in the multiplier algebra of $\mathcal{S}$ such that $\delta(a)=a x-x a$ for all operators $a \in \mathcal{S}$.

General references are: for functional analysis— [18], for Banach algebra theory — [7] and for non-Banach algebra theory-[14].

The author wishes to thank P. Domański for turning his attention to the question of selfderivations on the noncommutative Schwartz space and T. Ciaś for pointing out the result [25, Prop. 6.3.2].

\section{Notation and preliminaries}

Let

$$
s=\left\{\xi=\left(\xi_{j}\right)_{j \in \mathbb{N}} \subset \mathbb{C}^{\mathbb{N}}:|\xi|_{n}^{2}:=\sum_{j=1}^{+\infty}\left|\xi_{j}\right|^{2} j^{2 n}<+\infty \text { for all } n \in \mathbb{N}\right\}
$$

denote the so-called space of rapidly decreasing sequences. This space becomes Fréchet when endowed with the above defined sequence $\left(|\cdot|_{n}\right)_{n \in \mathbb{N}}$ of norms. It is isomorphic to the Schwartz space of test functions for tempered distributions or to the space of smooth functions on a compact, smooth manifold. The basis $\left(U_{n}\right)_{n \in \mathbb{N}}$ of zero neighbourhoods of $s$ is defined by $U_{n}:=\left\{\xi \in s:|\xi|_{n} \leqslant 1\right\}$. The topological dual of $s$ is the so-called space of slowly increasing sequences

$$
s^{\prime}=\left\{\eta=\left(\eta_{j}\right)_{j \in \mathbb{N}} \subset \mathbb{C}^{\mathbb{N}}:|\eta|_{-n}^{2}:=\sum_{j=1}^{+\infty}\left|\eta_{j}\right|^{2} j^{-2 n}<+\infty \text { for some } n \in \mathbb{N}\right\} .
$$

It is isomorphic to the space $\mathcal{S}^{\prime}\left(\mathbb{R}^{n}\right)$ of tempered distributions on $\mathbb{R}^{n}$ or to the space $\mathcal{E}^{\prime}(K)$ of distributions with support contained in a fixed, compact set $K \subset \mathbb{R}^{n}$.

The noncommutative Schwartz space $\mathcal{S}$ is the space $L\left(s^{\prime}, s\right)$ of all linear and continuous operators from the dual of $s$ into $s$ itself, endowed with the topology of uniform convergence on bounded sets. This is a Fréchet space topology described by the sequence $\left(\|\cdot\|_{n}\right)_{n \in \mathbb{N}}$ of norms given by

$$
\|x\|_{n}:=\sup \left\{|x \xi|_{n}: \quad \xi \in U_{n}^{\circ}\right\}
$$


where $U_{n}^{\circ}=\left\{\xi \in s^{\prime}:|\xi|_{n}^{\prime} \leqslant 1\right\}$ is the polar of the zero neighbourhood $U_{n} \subset s$. The identity map $\iota: s \hookrightarrow s^{\prime}$ is a continuous embedding and defines multiplication in $\mathcal{S}$ by

$$
x y:=x \circ \iota \circ y, \quad x, y \in \mathcal{S} .
$$

This multiplication is separately continuous therefore jointly continuous by [27, Th. 1.5]. Duality between $s$ and $s^{\prime}$ is given by

$$
\langle\xi, \eta\rangle:=\sum_{j \in \mathbb{N}} \xi_{j} \overline{\eta_{j}}, \quad \xi \in s, \eta \in s^{\prime}
$$

and it leads to an involution map on $\mathcal{S}$ defined by

$$
\left\langle x^{*} \xi, \eta\right\rangle:=\langle\xi, x \eta\rangle, \quad x \in \mathcal{S}, \xi, \eta \in s^{\prime} .
$$

With these operations the noncommutative Schwartz space $\mathcal{S}$ becomes an $m$-convex Fréchet *-algebra. It may be identified, via the *-algebra isomorphism $x \mapsto\left(\left\langle x e_{j}, e_{i}\right\rangle\right)_{i, j \in \mathbb{N}}$, $\left(e_{j}\right)_{j \in \mathbb{N}}$-standard unit vector basis, with the so-called algebra $\mathcal{K}^{\infty}$ of rapidly decreasing matrices defined as

$$
\mathcal{K}^{\infty}:=\left\{x=\left(x_{i, j}\right)_{i, j \in \mathbb{N}}:\|x\|_{n, \infty}:=\sup _{i, j \in \mathbb{N}}\left|x_{i j}\right|(i j)^{n}<+\infty \text { for all } n \in \mathbb{N}\right\} .
$$

The topological dual $\mathcal{S}^{\prime}$ may be therefore identified with the space of the so-called slowly increasing matrices, i.e.

$$
\mathcal{S}^{\prime}=\left\{\phi=\left(\phi_{i j}\right)_{i, j \in \mathbb{N}} \mid \sup \left\{\left|\phi_{i j}\right|(i j)^{-k}: i, j \in \mathbb{N}\right\}<+\infty \text { for some } k \in \mathbb{N}\right\} .
$$

The duality in the matrix language is given by the trace, i.e. if $x \in \mathcal{S}, \phi \in \mathcal{S}^{\prime}$ then

$$
\phi(x):=\langle\langle x, \phi\rangle\rangle=\sum_{i, j=1}^{+\infty} x_{i j} \overline{\phi_{i j}} .
$$

More on the dual space $\mathcal{S}^{\prime}$ may be found in [23]. The multiplier algebra $\mathcal{M S}$ of the noncommutative Schwartz space may be described in several ways, depending on the context-see [5, Corollary 4.4]. For our purposes the most convenient one will be to view the multiplier algebra as a *-algebra of all infinite scalar matrices $\left(x_{i j}\right)_{i, j \in \mathbb{N}}$ such that for every $N \in \mathbb{N}$ there is $n \in \mathbb{N}$ satisfying

$$
\|x\|_{N, n}:=\sup _{i, j \in \mathbb{N}}\left\{\left|x_{i j}\right| \cdot \max \left\{\frac{i^{N}}{j^{n}}, \frac{j^{N}}{i^{n}}\right\}\right\}<\infty .
$$

The natural topology on $\mathcal{M S}$ is that of a PLS-space. More precisely, there is a topological *-algebra isomorphism

$$
\mathcal{M S}=\operatorname{proj}_{N \in \mathbb{N}} \operatorname{ind}_{n \in \mathbb{N}} \ell_{\infty}\left(\left(a_{i j ; N, n}\right)_{i, j \in \mathbb{N}}\right),
$$

where

$$
a_{i j ; N, n}:=\max \left\{\frac{i^{N}}{j^{n}}, \frac{j^{N}}{i^{n}}\right\}
$$

and

$$
\ell_{\infty}\left(\left(a_{i j ; N, n}\right)_{i, j \in \mathbb{N}}\right):=\left\{\left(x_{i j}\right)_{i, j \in \mathbb{N}}: \sup _{i, j \in \mathbb{N}}\left|x_{i j}\right| a_{i j ; N, n}<\infty\right\}
$$


is a weighted $\ell_{\infty}$-space of bounded, doubly indexed sequences. It ia also useful to view $\mathcal{M S}$ as an algebra of all linear and continuous operators $x: s \rightarrow s$ which extend (necessarily uniquely) to linear and continuous operators $\tilde{x}: s^{\prime} \rightarrow s^{\prime}$. In other words, $\mathcal{M S}=L(s) \cap L\left(s^{\prime}\right)$ and the base of zero neighbourhoods in $\mathcal{M S}$ is given by the family $\{U \cap V\}$, where $U$, respectively $V$ runs through the base of zero neighbourhoods in $L(s)$, respectively in $L\left(s^{\prime}\right)$.

\section{Boundedly approximately contractible Fréchet algebras}

The notion of an amenable/contractible algebra admits a number of weakened versions see e.g. $[2,16]$. We will deal with the following one. A Fréchet algebra $A$ is boundedly approximately contractible if for every $A$-bimodule $X$ and every continuous derivation $\delta: A \rightarrow X$ there is a net $\left(x_{\alpha}\right)_{\alpha} \subset X$ such that

$$
\delta(a)=\lim _{\alpha}\left(a \cdot x_{\alpha}-x_{\alpha} \cdot a\right) \quad \forall a \in A
$$

and the set of inner derivations $\left(\operatorname{ad}_{x_{\alpha}}\right)_{\alpha} \subset L(A, X)$ is equicontinuous. By ad $\operatorname{ad}_{x}$ we denote the so-called inner derivation acting by $a \mapsto a \cdot x-x \cdot a, a \in A$. If $A$ is a unital algebra then by $\mathbf{1}$ we denote the unit element in $A$ and if $A$ is a non-unital algebra then by $A^{\#}$ we denote its unitization.

Proposition 1 Let A be a non-unital Fréchet algebra. Then A is boundedly approximately contractible if and only if $A^{\#}$ is boundedly approximately contractible.

Proof Sufficiency. Let $\delta: A \rightarrow X$ be a continuous derivation into an $A$-bimodule $X$. By defining $\delta(\mathbf{1}):=0$ and the module operations $\mathbf{1} \cdot x=x \cdot \mathbf{1}:=x, x \in X$, we get a continuous derivation $\delta: A^{\#} \rightarrow X$ into an $A^{\#}$-bimodule $X$. By assumption $\delta=\lim _{\alpha} \operatorname{ad}_{x_{\alpha}}$ and $\left(\operatorname{ad}_{x_{\alpha}}\right)_{\alpha} \subset L\left(A^{\#}, X\right)$ is equicontinuous. Since $A$ is a complemented ideal in $A^{\#}$, the net $\left(\operatorname{ad}_{x_{\alpha}}\right)_{\alpha}$ is also equicontinuous in $L(A, X)$.

Necessity. Let $\delta: A^{\#} \rightarrow X$ be a continuous derivation. By [16, Lemma 2.2] we may assume $X$ is unital, i.e. $\mathbf{1} \cdot x=x \cdot \mathbf{1}=x$ for all $x \in X$. Consequently, $\delta(\mathbf{1})=0$. Since $\left.\delta\right|_{A}$ is a continuous derivation, we get by assumption $\left.\delta\right|_{A}=\lim _{\alpha} \operatorname{ad}_{x_{\alpha}}$. Therefore

$$
\begin{aligned}
\delta(a+\lambda \mathbf{1})=\left(\left.\delta\right|_{A}\right)(a) & =\lim _{\alpha}\left(a \cdot x_{\alpha}-x_{\alpha} \cdot a+\lambda \mathbf{1} \cdot x_{\alpha}-x_{\alpha} \cdot \lambda \mathbf{1}\right) \\
& =\lim _{\alpha}\left((a+\lambda \mathbf{1}) \cdot x_{\alpha}-x_{\alpha} \cdot(a+\lambda \mathbf{1})\right)
\end{aligned}
$$

and the net $\left(\operatorname{ad}_{x_{\alpha}}\right)_{\alpha} \subset L\left(A^{\#}, X\right)$ is equicontinuous.

We are now going to give a characterization of boundedly approximately contractible Fréchet algebras. It was first stated (without proof) in [24]. Necessity in the case of Banach algebras was proved in [2, Th. 2.5]. The method of proof (sufficiency part) will be of particular importance for us. Before proceeding to this let us recall few basic definitions. If $X, Y$ are Fréchet spaces with the respective non-decreasing sequences of seminorms giving their topologies and $T: X \rightarrow Y$ is a linear and continuous operator then the characteristic of continuity of $T$ is a function $\sigma_{T}: \mathbb{N} \rightarrow \mathbb{N}$ such that for every $k \in \mathbb{N}, \sigma_{T}(k)$ is defined to be the smallest number $n$ satisfying

$$
\exists C>0 \forall x \in X:\|T x\|_{k} \leqslant C\|x\|_{n} .
$$


The topology of the projective tensor product $X \widehat{\otimes} Y$ is given by the sequence $\left(\|\cdot\|_{n}\right)_{n \in \mathbb{N}}$ of seminorms defined by

$$
\|u\|_{n}:=\inf \left\{\sum_{j=1}^{k}\left\|x_{j}\right\|_{n}\left\|y_{j}\right\|_{n}: u=\sum_{j=1}^{k} x_{j} \otimes y_{j}, x_{j} \in X, y_{j} \in Y, k \in \mathbb{N}\right\} .
$$

If $X, Y$ are algebras then

$$
\left(\sum_{i=1}^{m} x_{i} \otimes y_{i}\right) \cdot\left(\sum_{j=1}^{n} a_{j} \otimes b_{j}\right):=\sum_{i, j} x_{i} a_{j} \otimes y_{i} b_{j}
$$

and if they both are involutive then

$$
\left(\sum_{i=1}^{m} x_{i} \otimes y_{i}\right)^{*}:=\sum_{i=1}^{m} x_{i}^{*} \otimes y_{i}^{*}
$$

If $A$ is a Fréchet algebra then the product map $\pi: A \widehat{\otimes} A \rightarrow A$ is defined by

$$
\pi\left(\sum_{j=1}^{k} a_{j} \otimes b_{j}\right):=\sum_{j=1}^{k} a_{j} b_{j}
$$

and extended by density. With respect to the projective tensor product topology, the product map is continuous.

Theorem 1 Let A be a Fréchet algebra with the topology given by anon-decreasing sequence $\left(\|\cdot\|_{k}\right)_{k \in \mathbb{N}}$ of seminorms. TFAE:

(1) A is boundedly approximately contractible,

(2) there are nets $\left(M_{\alpha}\right)_{\alpha} \subset A \widehat{\otimes} A,\left(F_{\alpha}\right)_{\alpha},\left(G_{\alpha}\right)_{\alpha} \subset A$, a function $\sigma: \mathbb{N} \rightarrow \mathbb{N}$ and a sequence $\left(C_{k}\right)_{k}$ of positive constants satisfying:

(i) $\pi\left(M_{\alpha}\right)=F_{\alpha}+G_{\alpha}$ for all $\alpha$,

(ii) $\lim _{\alpha} a F_{\alpha}=\lim _{\alpha} G_{\alpha} a=$ a for all $a \in A$,

(iii) $\left\|a F_{\alpha}\right\|_{k} \leqslant C_{k}\|a\|_{\sigma(k)},\left\|G_{\alpha} a\right\|_{k} \leqslant C_{k}\|a\|_{\sigma(k)}$ for all $\alpha$, all $a \in A$ and all $k \in \mathbb{N}$,

(iv) $\lim _{\alpha}\left(a \cdot M_{\alpha}-M_{\alpha} \cdot a-a \otimes G_{\alpha}+F_{\alpha} \otimes a\right)=0$ for all $a \in A$,

(v) $\left\|a \cdot M_{\alpha}-M_{\alpha} \cdot a-a \otimes G_{\alpha}+F_{\alpha} \otimes a\right\|_{k} \leqslant C_{k}\|a\|_{\sigma(k)}$ for all $\alpha$, all $a \in A$ and all $k \in \mathbb{N}$.

Proof $(1) \Rightarrow(2)$ : this is similar to the proof of [2, Th. 2.5].

$(2) \Rightarrow(1)$ : by Proposition 1 we may assume $A$ is unital. Define

$$
d_{\alpha}:=M_{\alpha}-F_{\alpha} \otimes \mathbf{1}-\mathbf{1} \otimes G_{\alpha}+\mathbf{1} \otimes \mathbf{1} \in A \widehat{\otimes} A
$$

and observe that

$$
\begin{aligned}
a \cdot d_{\alpha}-d_{\alpha} \cdot a= & \left(a \cdot M_{\alpha}-M_{\alpha} \cdot a-a \otimes G_{\alpha}+F_{\alpha} \otimes a\right) \\
& +\left(a-a F_{\alpha}\right) \otimes \mathbf{1}+\mathbf{1} \otimes\left(G_{\alpha} a-a\right) \rightarrow 0 .
\end{aligned}
$$

Moreover,

$$
\left\|a \cdot d_{\alpha}-d_{\alpha} \cdot a\right\|_{k} \leqslant 3 C_{k}\|a\|_{\sigma(k)} \quad \forall \alpha .
$$

Using assumption (i) we obtain

$$
\pi\left(d_{\alpha}\right)=\pi\left(M_{\alpha}\right)-F_{\alpha}-G_{\alpha}+\mathbf{1}=\mathbf{1} \quad \forall \alpha .
$$


Let now $\delta: A \rightarrow X$ be a continuous derivation into a unital $A$-bimodule $X$ (this restriction is justified by [16, Lemma 2.2]). Define

$$
D: A \widehat{\otimes} A \rightarrow X, \quad D(a \otimes b):=a \cdot \delta(b)
$$

and extend linearly. Clearly, $D$ is continuous. Put

$$
x_{\alpha}:=D\left(d_{\alpha}\right)
$$

and observe that if we denote

$$
d_{\alpha}=\sum_{n=1}^{\infty} a_{n}^{\alpha} \otimes b_{n}^{\alpha}
$$

then

$$
x_{\alpha}=\sum_{n=1}^{\infty} a_{n}^{\alpha} \cdot \delta\left(b_{n}^{\alpha}\right) .
$$

Now we choose an arbitrary element $a \in A$ and make the following computation:

$$
\begin{aligned}
a \cdot x_{\alpha}-x_{\alpha} \cdot & a-\delta(a) \\
= & \sum_{n} a a_{n}^{\alpha} \cdot \delta\left(b_{n}^{\alpha}\right)-\sum_{n} a_{n}^{\alpha} \cdot \delta\left(b_{n}^{\alpha}\right) \cdot a-\sum_{n} a_{n}^{\alpha} b_{n}^{\alpha} \cdot \delta(a) \\
= & \sum_{n} a a_{n}^{\alpha} \cdot \delta\left(b_{n}^{\alpha}\right)-\sum_{n} a_{n}^{\alpha} \cdot \delta\left(b_{n}^{\alpha} a\right) \\
= & D\left(a \cdot d_{\alpha}-d_{\alpha} \cdot a\right) .
\end{aligned}
$$

The first equality follows by (10) and the fact that $X$ is unital while the second equality follows by the derivation rule. Continuity of $D$ and (8) imply

$$
\delta(a)=\lim _{\alpha}\left(a \cdot x_{\alpha}-x_{\alpha} \cdot a\right) \quad \forall a \in A .
$$

Equicontinuity of $\left(\operatorname{ad}_{x_{\alpha}}\right)_{\alpha} \subset L(A, X)$ follows by (9). Indeed, for every $k \in \mathbb{N}$ we have

$$
\begin{aligned}
\left\|a \cdot x_{\alpha}-x_{\alpha} \cdot a\right\|_{k} & \leqslant\|\delta(a)\|_{k}+\left\|D\left(a \cdot d_{\alpha}-d_{\alpha} \cdot a\right)\right\|_{k} \\
& \leqslant A_{k}\|a\|_{\sigma_{\delta}(k)}+3 B_{k} C_{k}\|a\|_{\sigma\left(\sigma_{D}(k)\right)}
\end{aligned}
$$

and the constants $A_{k}, B_{k}$ satisfy (7) for $\delta: A \rightarrow X$ and $D: A \widehat{\otimes} A \rightarrow X$, respectively.

\section{Self-derivations on $\mathcal{S}$}

We start by making several observations. Let $\left(u_{n}\right)_{n \in \mathbb{N}} \subset \mathcal{S}$ be a sequence of operators in the noncommutative Schwartz space defined by

$$
u_{n}: s^{\prime} \rightarrow s, \quad u_{n}\left(\left(\xi_{j}\right)_{j}\right):=\left(\xi_{1}, \ldots \xi_{n}, 0,0, \ldots\right) .
$$

We can also view each $u_{n}$ as an infinite matrix

$$
u_{n}=\left(\begin{array}{cc}
I_{n} & 0 \\
0 & 0
\end{array}\right)
$$

where $I_{n}$ is the $n \times n$ identity matrix. From [22, Prop. 2] it follows that $\left(u_{n}\right)_{n}$ is a sequential approximate identity. 
If $\delta$ is a derivation on a 'matrix algebra' then it has to have a specific form on matrix units $\left(e_{i j}\right)_{i, j \in \mathbb{N}}$. Indeed, since

$$
\delta\left(e_{i j}\right)=\delta\left(e_{i j}\right) e_{j j}+e_{i j} \delta\left(e_{j j}\right)=e_{i i} \delta\left(e_{i j}\right)+\delta\left(e_{i i}\right) e_{i j},
$$

the matricial form of $\delta\left(e_{i j}\right)=\left(t_{p q}^{i j}\right)_{p, q \in \mathbb{N}}$ is

$$
\left(\begin{array}{cccccc} 
& & & t_{1 j}^{i j} & & \\
& & & \vdots & \mathbf{0} & \\
& & & t_{i-1, j}^{i j} & & \\
t_{j 1}^{j j} & \ldots & t_{j, j-1}^{j j} & t_{i j}^{i j} & t_{j, j+1}^{j j} & \ldots \\
& & & t_{i+1, j}^{i j} & & \\
& & & \vdots & \mathbf{0} &
\end{array}\right.
$$

where the only non-zero entries lie in the $i$-th row and $j$-th column. Observe that in the $(i, j)$-th entry the 'missing' summand $t_{j j}^{j j}$ (on the left) and $t_{i i}^{i i}$ (on the right) is zero. Indeed, $e_{k k}$ is a projection for every $k \in \mathbb{N}$ therefore by [7, Prop. 1.8.2]

$$
t_{k k}^{k k}=\left\langle\left\langle e_{k k}, \delta\left(e_{k k}\right)\right\rangle\right\rangle=\left\langle\left\langle e_{k k}, e_{k k} \delta\left(e_{k k}\right) e_{k k}\right\rangle\right\rangle=0 .
$$

From this we obtain that $\delta\left(u_{n}\right)$ is of the form

$$
\left(\begin{array}{cccccc} 
& & & t_{1, n+1}^{11} & t_{1, n+2}^{11} & \cdots \\
& \mathbf{0} & & \vdots & \vdots & \ldots \\
& & & t_{n, n+1}^{n n} & t_{n, n+2}^{n n} & \cdots \\
t_{n+1,1}^{11} & \ldots & t_{n+1, n}^{n n} & & & \\
t_{n+2,1}^{11} & \ldots & t_{n+2, n}^{n n} & & & \\
\vdots & \vdots & \vdots & & \mathbf{0} &
\end{array}\right) .
$$

Observe that by [21, Prop. 2] we have an $n \times n$ zero matrix in the upper left corner. The last matrix we are interested in is $\frac{1}{n} \sum_{i, j=1}^{n} e_{j i} \delta\left(e_{i j}\right)$. It is of the form

$$
\left(\begin{array}{ccccccc}
0 & t_{12}^{11} & t_{13}^{11} & \ldots & t_{1 n}^{11} & t_{1, n+1}^{11} & \ldots \\
-t_{21}^{11} & 0 & t_{23}^{22} & \ldots & t_{2 n}^{22} & t_{2, n+1}^{22} & \ldots \\
-t_{31}^{11} & -t_{32}^{22} & 0 & \ldots & t_{3 n}^{33} & t_{3, n+1}^{33} & \ldots \\
\vdots & \vdots & \vdots & \ddots & \vdots & \vdots & \\
-t_{n 1}^{11} & -t_{n 2}^{22} & -t_{n 3}^{33} & \ldots & 0 & t_{n, n+1}^{n n} & \ldots \\
& & & & & &
\end{array}\right)
$$

Observe that the upper triangular entries follow by adding rows appearing on the left hand side of (12) while the lower ones follow by adding columns appearing on the right hand side of (12). This is indeed the case, since it follows from (13) that for any $p, q=1, \ldots, n$ we have

$$
\left\langle\left\langle e_{p q}, \frac{1}{n} \sum_{i, j=1}^{n}\left(e_{j i} \delta\left(e_{i j}\right)+\delta\left(e_{j i}\right) e_{i j}\right)\right\rangle\right\rangle=\left\langle\left\langle e_{p q}, \delta\left(u_{n}\right)\right\rangle\right\rangle=0 .
$$


Before proceeding to the main result of the paper we will need the following fact.

Proposition 2 The multiplication map $M: \mathcal{S} \times \mathcal{M S} \rightarrow \mathcal{S}$ is separately continuous.

Proof Fix $y \in \mathcal{M S}$ and define the map $M_{y}: \mathcal{S} \rightarrow \mathcal{S}$ by $M_{y}(x):=x y$. To show its continuity, let $N \in \mathbb{N}$. By (5) there is $n \in \mathbb{N}$ such that $\|y\|_{N, n}<\infty$ (recall that we can always take $n \geqslant N)$. This leads to the following estimations:

$$
\begin{aligned}
\|x y\|_{N, \infty} & \leqslant \sup _{i, j \in \mathbb{N}} \sum_{k=1}^{\infty}\left|x_{i k}\right|\left|y_{k j}\right|(i j)^{N} \\
& \leqslant \sup _{i, j \in \mathbb{N}} \sum_{k=1}^{\infty} k^{-2}\left|x_{i k}\right|(i k)^{n+2}\left|y_{k j}\right| \frac{j^{N}}{k^{n}} \\
& \leqslant C\|y\|_{N, n}\|x\|_{n+2, \infty},
\end{aligned}
$$

where we have denoted $C:=\sum_{k} k^{-2}$. On the other hand, for a fixed $x \in \mathcal{S}$ we define the map $M_{x}: \mathcal{M S} \rightarrow \mathcal{S}$ by $M_{x}(y):=x y$. Since by (6) we have

$$
\mathcal{M S}=\operatorname{proj}_{N \in \mathbb{N}} \operatorname{ind}_{n \in \mathbb{N}} \ell_{\infty}\left(\left(a_{i j ; N, n}\right)_{i, j \in \mathbb{N}}\right),
$$

continuity of $M_{x}$ means that (see [18, Propositions 22.6, 24.7]) for every $N \in \mathbb{N}$ there is $M \in \mathbb{N}$ such that for any $m \in \mathbb{N}$ there exists $C>0$ satisfying

$$
\|x y\|_{N, \infty} \leqslant C\|y\|_{M, m} \quad(y \in \mathcal{M S}) .
$$

Following the computations for the map $M_{y}$ above we get

$$
\|x y\|_{N, \infty} \leqslant C\|x\|_{m+2, \infty}\|y\|_{M, m} \quad(y \in \mathcal{M S})
$$

with the same constant $C=\sum_{k} k^{-2}$.

Now we are ready to characterize self-derivations on the noncommutative Schwartz space. Recall from [22, Theorem 13] that every such derivation is continuous.

Theorem 2 If $\delta: \mathcal{S} \rightarrow \mathcal{S}$ is a derivation on the noncommutative Schwartz space then there exists an element $x$ in the multiplier algebra $\mathcal{M S}$ of the noncommutative Schwartz space such that

$$
\delta(a)=a x-x a \quad \forall a \in \mathcal{S} .
$$

Proof From [22, Th. 16] it follows that the noncommutative Schwartz space satisfies condition (2) of Theorem 1 with the following sequences:

$$
M_{n}:=u_{n} \otimes u_{n}+\frac{1}{n} \sum_{i, j=1}^{n} e_{i j} \otimes e_{j i}, F_{n}=G_{n}=u_{n}, \sigma(k)=3 k+1, C_{k}=4 .
$$

Let now $\delta: \mathcal{S} \rightarrow \mathcal{S}$ be a derivation. By (11) and the proof of Theorem 1 we can observe that

$$
\delta(a)=\lim _{n \rightarrow \infty}\left(a x_{n}-x_{n} a\right) \quad \forall a \in \mathcal{S},
$$

where

$$
x_{n}=u_{n} \delta\left(u_{n}\right)-\delta\left(u_{n}\right)+\frac{1}{n} \sum_{i, j=1}^{n} e_{j i} \delta\left(e_{i j}\right) \in \mathcal{S} .
$$


If we now denote $\delta\left(e_{i j}\right)=\left(t_{p q}^{i j}\right)_{p, q \in \mathbb{N}}$ then by (13) and (14) we get that

$$
x_{n}=\left(\begin{array}{ccccccc}
0 & t_{12}^{11} & t_{13}^{11} & \ldots & t_{1 n}^{11} & t_{1, n+1}^{11} & \ldots \\
-t_{21}^{11} & 0 & t_{23}^{22} & \ldots & t_{2 n}^{22} & t_{2, n+1}^{22} & \ldots \\
-t_{31}^{11} & -t_{32}^{22} & 0 & \ldots & t_{3 n}^{33} & t_{3, n+1}^{33} & \ldots \\
\vdots & \vdots & \vdots & \ddots & \vdots & \vdots & \\
-t_{n 1}^{11} & -t_{n 2}^{22} & -t_{n 3}^{33} & \ldots & 0 & t_{n, n+1}^{n n} & \ldots \\
-t_{n+1,1}^{11} & -t_{n+1,2}^{22} & -t_{n+1,3}^{33} & \ldots & -t_{n+1, n}^{n n} & & \\
-t_{n+2,1}^{11} & -t_{n+2,2}^{22} & -t_{n+2,3}^{33} & \ldots & -t_{n+2, n}^{n n} & & \\
\vdots & \vdots & \vdots & \vdots & \vdots & 0 &
\end{array}\right) .
$$

In general, the sequence $\left(x_{n}\right)_{n \in \mathbb{N}} \subset \mathcal{S}$ need not be bounded in $\mathcal{S}$. We will show thathowever-it is bounded in (the weaker topology of) the multiplier algebra $\mathcal{M S}$. To this end, observe that continuity of $\delta$ implies that

$$
\forall K \in \mathbb{N} \exists k \in \mathbb{N}, C_{K}>0 \forall x \in \mathcal{S}: \quad\|\delta(x)\|_{K, \infty} \leqslant C_{K}\|x\|_{k, \infty}
$$

(continuity with respect to the topology given by the norms defined in (3)). Applying this to matrix units $\left(e_{i j}\right)_{i, j \in \mathbb{N}}$ we get

$$
\forall K \in \mathbb{N} \exists k \in \mathbb{N}, C_{K}>0 \forall i, j, p, q \in \mathbb{N}: \quad\left|t_{p q}^{i j}\right| \leqslant C_{K} \frac{(i j)^{k}}{(p q)^{K}} .
$$

Consequently, for any $i, j \in \mathbb{N}$ this implies

$$
\left|t_{i j}^{i i}\right| \leqslant C_{K} \frac{i^{2 k}}{(i j)^{K}} \leqslant C_{K} \frac{i^{2 k}}{j^{K}}
$$

and

$$
\left|t_{i j}^{j j}\right| \leqslant C_{K} \frac{j^{2 k}}{(i j)^{K}} \leqslant C_{K} \frac{j^{2 k}}{i^{K}} .
$$

We can now estimate entries of the matrices $x_{n}, n \in \mathbb{N}$. From (16) we know that

$$
\left\langle\left\langle e_{i j}, x_{n}\right\rangle\right\rangle= \begin{cases}t_{i j}^{i i}, & \text { if } i<j \text { and } \mathrm{i} \leqslant \mathrm{n} \\ -t_{i j}^{j j}, & \text { if } i>j \text { and } \mathrm{j} \leqslant \mathrm{n} \\ 0, & \text { if } i \geqslant n \text { and } \mathrm{j} \geqslant \mathrm{n}\end{cases}
$$

and for any $K, k \in \mathbb{N}$

$$
\max \left\{\frac{i^{K}}{j^{k}}, \frac{j^{K}}{i^{k}}\right\}=\left\{\begin{array}{ll}
\frac{j^{K}}{i^{k}}, & \text { if } i<j \\
\frac{i^{K}}{j^{k}}, & \text { if } i>j
\end{array} .\right.
$$

Therefore for every $n \in \mathbb{N}$ and any $i, j \in \mathbb{N}$ we obtain that

$$
\left|\left\langle\left\langle e_{i j}, x_{n}\right\rangle\right\rangle\right| \max \left\{\frac{i^{K}}{j^{2 k}}, \frac{j^{K}}{i^{2 k}}\right\} \leqslant C_{K} .
$$


Using the notation from p. 3 we may write that for every $K \in \mathbb{N}$ there is $k \in \mathbb{N}$ such that $\left(x_{n}\right)_{n \in \mathbb{N}}$ is a bounded sequence in $\ell_{\infty}\left(\left(a_{i j ; K, 2 k}\right)_{i, j \in \mathbb{N}}\right)$. By [18, Lemma 24.2] $\left(x_{n}\right)_{n}$ is bounded in $\mathcal{M S}$. Since the multiplier algebra is nuclear by [5, Cor. 4.2], it then follows (see [18, Corollary 28.5, Lemma 24.19]) that the sequence $\left(x_{n}\right)_{n}$ has a convergent subsequence, say $x:=\lim _{n} x_{k_{n}}$. Now we wish to apply (15). The only point is that the limit in (15) is taken in $\mathcal{S}$ while $\left(x_{k_{n}}\right)_{n}$ tends to $x$ in the (weaker) topology of $\mathcal{M S}$. Observe, however, that by Proposition 2 the multiplication map $M: \mathcal{S} \times \mathcal{M S} \rightarrow \mathcal{S}$ is separately continuous. Consequently,

$$
\delta(a)=a x-x a \quad \forall a \in \mathcal{S} .
$$

Alternatively (without involving nuclearity of the multiplier algebra) one might wish to find the explicit form of the limit operator $x \in \mathcal{M S}$. By (16) we can observe that $\lim _{n} x_{n}=x$, where

$$
x=\left(\begin{array}{cccc}
0 & t_{12}^{11} & t_{13}^{11} & \ldots \\
-t_{21}^{11} & 0 & t_{23}^{22} & \ldots \\
-t_{31}^{11} & -t_{32}^{22} & 0 & \ldots \\
\vdots & \vdots & \vdots & \ddots
\end{array}\right)
$$

Indeed,

$$
\left\langle\left\langle e_{i j}, x-x_{n}\right\rangle\right\rangle= \begin{cases}t_{i j}^{i i}, & \text { if } n<i<j \\ -t_{i j}^{j j}, & \text { if } n<j<i \\ 0, & \text { if } i \leqslant n \text { or } j \leqslant n \text { or } i=j\end{cases}
$$

and by (17) we obtain

$$
\sup _{i, j \in \mathbb{N}}\left\{\left|\left\langle\left\langle e_{i j}, x-x_{n}\right\rangle\right\rangle\right| \max \left\{\frac{i^{K}}{j^{2 k+1}}, \frac{j^{K}}{i^{2 k+1}}\right\}\right\} \leqslant C_{K} \frac{1}{n} \rightarrow 0 .
$$

In other words, for every $K \in \mathbb{N}$ there is another $k \in \mathbb{N}$ so that $\lim _{n}\left\|x-x_{n}\right\|_{K, k}=0$ which gives exactly convergence of a sequence in a PLS-space.

Acknowledgements The research of the author has been supported in the years 2014-2017 by the National Center of Science, Poland, Grant No. UMO-2013/10/A/ST1/00091.

Open Access This article is distributed under the terms of the Creative Commons Attribution 4.0 International License (http://creativecommons.org/licenses/by/4.0/), which permits unrestricted use, distribution, and reproduction in any medium, provided you give appropriate credit to the original author(s) and the source, provide a link to the Creative Commons license, and indicate if changes were made.

\section{References}

1. Bhatt, S.J., Inoue, A., Ogi, H.: Spectral invariance, $K$-theory isomorphism and an application to the differential structure of $C^{*}$-algebras. J. Oper. Theory 49(2), 389-405 (2003)

2. Choi, Y., Ghahramani, F., Zhang, Y.: Approximate and pseudo-amenability of various classes of Banach algebras. J. Funct. Anal. 256(10), 3158-3191 (2009)

3. Ciaś, T.: Commutative subalgebras of the algebra of smooth operators. Monatsh. Math. 181(2), 301-323 (2016)

4. Ciaś, T.: On the algebra of smooth operators. Stud. Math. 218(2), 145-166 (2013)

5. Ciaś, T., Piszczek, K.: The multiplier algebra of the noncommutative Schwartz space. Banach J. Math. Anal. (To appear) 
6. Cuntz, J.: Cyclic theory and the bivariant Chern-Connes character. In: Noncommutative Geometry, Lecture Notes in Math., vol. 1831, pp. 73-135. Springer, Berlin (2004)

7. Dales, H.G.: Banach algebras and automatic continuity, In: London Mathematical Society Monographs. New Series, vol. 24. The Clarendon Press Oxford University Press, New York (2000) (Oxford Science Publications)

8. Domański, P.: Algebra of smooth operators. http://main3.amu.edu.pl/ domanski/salgebra1 (posted 11.01.2012)

9. Dubin, D.A., Hennings, M.A.: Quantum mechanics, algebras and distributions, Pitman Research Notes in Mathematics Series. vol. 238. Longman Scientific \& Technical, Harlow; copublished in the United States with John Wiley \& Sons, Inc., New York (1990)

10. Dubois-Violette, M., Kriegl, A., Maeda, Y., Michor, P. W.: Smooth *-algebras. Progr. Theor. Phys. Suppl. (144), 54-78 (2001) (Noncommutative geometry and string theory (Yokohama, 2001))

11. Effros, E.G., Webster, C.: Operator analogues of locally convex spaces. In: Operator Algebras and Applications (Samos, 1996), NATO Adv. Sci. Inst. Ser. C Math. Phys. Sci., vol. 495, pp. 163-207. Kluwer Academic Publishers, Dordrecht (1997)

12. Effros, E.G., Winkler, S.: Matrix convexity: operator analogues of the bipolar and Hahn-Banach theorems. J. Funct. Anal. 144(1), 117-152 (1997)

13. Elliott, G.A., Natsume, T., Nest, R.: Cyclic cohomology for one-parameter smooth crossed products. Acta Math. 160(3-4), 285-305 (1988)

14. Fragoulopoulou, M.: Topological algebras with involution. In: North-Holland Mathematics Studies, vol. 200. Elsevier Science B.V, Amsterdam (2005)

15. Glöckner, H., Langkamp, B.: Topological algebras of rapidly decreasing matrices and generalizations. Topol. Appl. 159(9), 2420-2422 (2012)

16. Lawson, P., Read, C.J.: Approximate amenability of Fréchet algebras. Math. Proc. Camb. Philos. Soc. 145(2), 403-418 (2008)

17. Levene, R.H., Piszczek, K.: Grothendieck's inequality in the noncommutative Schwartz space. Stud. Math. 234(2), 185-194 (2016)

18. Meise, R., Vogt, D.: Introduction to Functional Analysis, In: Oxford Graduate Texts in Mathematics, vol. 2, The Clarendon Press Oxford University Press, New York (1997) (Translated from the German by M. S. Ramanujan and revised by the authors)

19. Phillips, N.Ch.: $K$-theory for Fréchet algebras. Int. J. Math. 2(1), 77-129 (1991)

20. Pirkovskii, A.Yu.: Flat cyclic Fréchet modules, amenable Fréchet algebras, and approximate identities. Homol. Homot. Appl. 11(1), 81-114 (2009)

21. Piszczek, K.: The noncommutative Schwartz space is weakly amenable. Glasgow Math. J. doi:10.1017/ S0017089516000264 (To appear)

22. Piszczek, K.: Automatic continuity and amenability in the non-commutative Schwartz space. J. Math. Anal. Appl. 432(2), 954-964 (2015)

23. Piszczek, K.: A Jordan-like decomposition in the noncommutative Schwartz space. Bull. Aust. Math. Soc. 91(2), 322-330 (2015)

24. Piszczek, K.: Corrigendum to "Automatic continuity and amenability in the non-commutative Schwartz space”. J. Math. Anal. Appl. 435(1), 1015-1016 (2016)

25. Schmüdgen, K.: Unbounded operator algebras and representation theory. In: Operator Theory: Advances and Applications, vol. 37. Birkhäuser, Basel (1990)

26. Schweitzer, L.B.: Spectral invariance of dense subalgebras of operator algebras. Int. J. Math. 4(2), 289-317 (1993)

27. Żelazko, W., Selected topics in topological algebras. Lectures 1969/1970. Lecture Notes Series, No. 31. Matematisk Institut, Aarhus Universitet, Aarhus (1971) 